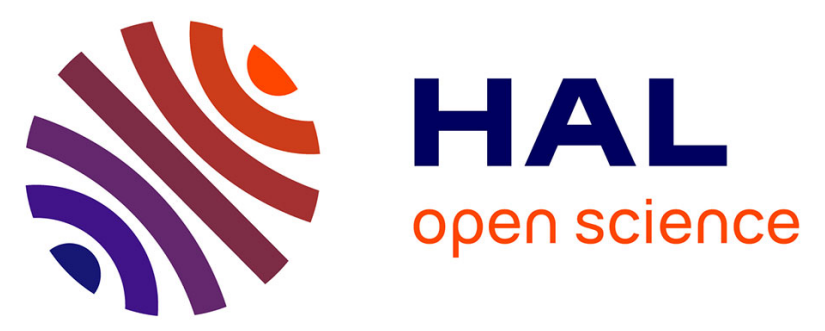

\title{
Comparison of prompt and delayed photofission neutron detection techniques using different types of radiation detectors
}

P. Sibczynski, A. Dziedzic, K. Grodzicki, J. Iwanowska, T. Kosinski, M. Matusiak, M. Moszynski, L. Swiderski, A. Syntfeld-Kazuch, D. Wolski, et al.

\section{To cite this version:}

P. Sibczynski, A. Dziedzic, K. Grodzicki, J. Iwanowska, T. Kosinski, et al.. Comparison of prompt and delayed photofission neutron detection techniques using different types of radiation detectors. 2016 IEEE Nuclear Science Symposium, Medical Imaging Conference and Room-Temperature Semiconductor Detector Workshop (NSS/MIC/RTSD), Oct 2016, Strasbourg, France. pp.8069697, 10.1109/NSSMIC.2016.8069697 . cea-01809224

\section{HAL Id: cea-01809224 https://hal-cea.archives-ouvertes.fr/cea-01809224}

Submitted on 4 Feb 2019

HAL is a multi-disciplinary open access archive for the deposit and dissemination of scientific research documents, whether they are published or not. The documents may come from teaching and research institutions in France or abroad, or from public or private research centers.
L'archive ouverte pluridisciplinaire HAL, est destinée au dépôt et à la diffusion de documents scientifiques de niveau recherche, publiés ou non, émanant des établissements d'enseignement et de recherche français ou étrangers, des laboratoires publics ou privés. 


\title{
Comparison of prompt and delayed photofission neutron detection techniques using different types of radiation detectors
}

\author{
Pawel Sibczynski, Andrzej Dziedzic, Krystian Grodzicki, Joanna Iwanowska, Tymoteusz Kosiński, \\ Michał Matusiak, Marek Moszyński, Lukasz Swiderski, Agnieszka Syntfeld-Każuch, Dariusz Wolski, \\ Frédérick Carrel, Amélie Grabowski, Matthieu Hamel, Frederic Lainé, Adrien Sari, Alessandro Iovene, \\ Carlo Tintori
}

\begin{abstract}
For several years, detection of various threats on country borders plays a significant role in the frame of Homeland Security applications. One of this threat is the illicit trafficking of nuclear materials (especially including Special Nuclear Material - SNM - ${ }^{235} \mathbf{U},{ }^{233} \mathbf{U}$ or ${ }^{239} \mathbf{P u}$ ), which can be potentially used for production of nuclear weapon as well as radiological dispersal device (RDD) - known also as a "dirty bomb". In order to detect the potentially hidden nuclear material, systems using linear accelerators and a group of detectors are developed by several scientific groups around the world. Besides solutions focusing on detection of delayed $\gamma$-rays or neutrons, also the systems dedicated for prompt neutron detection were proposed. One of the possible prompt neutron detection technique is known as Threshold Activation Detection (TAD). This technique relies on activation of ${ }^{19} F$ nuclei in the scintillator medium by fast neutrons and registration of high-energy $\beta$ particles and $\gamma$-rays from the decay of reaction products (for example, ${ }^{19} \mathbf{F}(\mathbf{n}, \alpha){ }^{16} \mathbf{N}$ or $\left.{ }^{19} \mathbf{F}(\mathbf{n}, \mathbf{p})^{19} \mathbf{O}\right)$. Recent studies in the frame of the European Horizon 2020 C-BORD project showed that, despite the low ${ }^{19} \mathbf{F}(\mathbf{n}, \alpha){ }^{16} \mathbf{N}$ or ${ }^{19} \mathbf{F}(\mathbf{n}, \mathbf{p}){ }^{19} \mathbf{O}$ reaction cross-section, the method could be a good solution for detection of shielded nuclear material. A benchmark of the TAD technique based on fluorine detectors with reference method focused on delayed neutron detection with ${ }^{3} \mathrm{He}$ detectors will be presented in this paper. These experimental results were obtained using $9 \mathrm{MeV}$ Varian Linatron M9 linear accelerator (LINAC).
\end{abstract}

Index Terms-photofission, prompt neutrons, $\mathrm{BaF}_{2}$, delayed neutrons, threshold activation detection, TAD, LINAC, special nuclear materials, SNM, scintillators.

\section{INTRODUCTION}

$\mathbf{T}$ HE aim of the study is to carry out a comparative study of nuclear material detection techniques basing on prompt and delayed neutron registration. The first technique,

This project has received funding from the European Union's Horizon 2020 research and innovation programme under grant agreement No 653323 . Disclaimer: This text reflects only the author's views and the Commission is not liable for any use that may be made of the information contained therein

Pawel Sibczynski, Andrzej Dziedzic, Krystian Grodzicki, Joanna Iwanowska, Tymoteusz Kosiński, Michał Matusiak, Marek Moszyński, Lukasz Swiderski, Agnieszka Syntfeld-Każuch, Dariusz Wolski are with the National Centre for Nuclear Research (NCBJ), Otwock, 05-400 Poland (telephone: +48 22273 1440, e-mail: pawel.sibczynski@ncbj.gov.pl, pawel.sibczynski@gmail.com)

Frédérick Carrel, Amélie Grabowski, Matthieu Hamel, Frederic Lainé, Adrien Sari are with CEA, LIST, Sensors and Electronic Architectures Laboratory, F-91191 Gif-sur-Yvette, France.

Alessandro Iovene, Carlo Tintori are with the CAEN S.p.A., Via Vetraia 11, 55049 - Viareggio (LU) - Italy. applied for prompt neutron detection, is based on Threshold Activation Detection (TAD) using scintillators containing ${ }^{19} \mathrm{~F}$. The incident fast neutrons activate the ${ }^{19} \mathrm{~F}$ nuclei, resulting in $(\mathrm{n}, \alpha)$ or $(\mathrm{n}, \mathrm{p})$ reaction and emission of high energy $\beta$ particles (up to $10.4 \mathrm{MeV}$ ) and $\gamma$-rays (in example 6.1 MeV). Potential scintillators of interest for this application are liquid fluorocarbon based EJ-313 [1], [2], [3] or BC-508, $\mathrm{BaF}_{2}$ [4] or fluorine based plastic scintillators based on pentafluorostyrene [5]. The second technology is focused on the detection of delayed neutrons using ${ }^{3} \mathrm{He}$ detectors [6], having a very low sensitivity to $\gamma$-rays and high cross section for thermal neutrons of 5530 barns. In this paper we investigated the performance of $\varnothing 5 " \times 1 " \mathrm{BaF}_{2}$ scintillator and compared with that for ${ }^{3} \mathrm{He}$ detector, Canberra $150 \mathrm{NH} 100$ model [7]. Particularly, $\mathrm{BaF}_{2}$ scintillators present many advantages comparing to fluorocarbon liquid scintillators, such as non-flammability and lack of hydrogen in the scintillator medium, decreasing the efficiency of threshold reaction due to neutron scattering. In addition, the risk of leakage of the liquid fluorocarbon scintillators is a significant disadvantage, promoting the $\mathrm{BaF}_{2}$ scintillator as an interesting alternative.

\section{RESULTS}

\section{A. Measurements of the $\mathrm{BaF}_{2}$ response to the ${ }^{252} \mathrm{Cf}$}

The $\mathrm{BaF}_{2}$ scintillator was coupled to an Electron Tubes ET9390 series $\varnothing 130 \mathrm{~mm}$ photomultiplier with silicone grease in order to improve light transportation to a photocathode. Finally, detector was mounted into laboratory-made housing designed and manufactured at NCBJ. The test site at NCBJ, where the detector was exposed to neutrons from a ${ }^{252} \mathrm{Cf}$ source is presented in Fig. 1.

Primarily, the response of the $\mathrm{BaF}_{2}$ to neutrons was measured for ${ }^{252} \mathrm{Cf}$ neutron source at NCBJ, emitting 30000 neutrons per second in $4 \pi$. Source was shielded with $5 \mathrm{~cm}$ thick $\mathrm{Pb}$ brick in order to absorb high-energy prompt and delayed $\gamma$-rays. The ${ }^{252} \mathrm{Cf}$ was placed $15 \mathrm{~cm}$ from the front of the detector. Anode pulses from the photomultiplier were recorded by CAEN V1730 VME-type digitizer offering sampling rate of $500 \mathrm{MS} / \mathrm{s}$ and accuracy of 14 bits. Pulses were integrated by a DPP-PSD firmware implemented to the digitizer FPGA. The net spectrum recorded after $4 \mathrm{~h}$ was showed in Fig. 2 . It can be noticed that fast neutron detection efficiency of the 


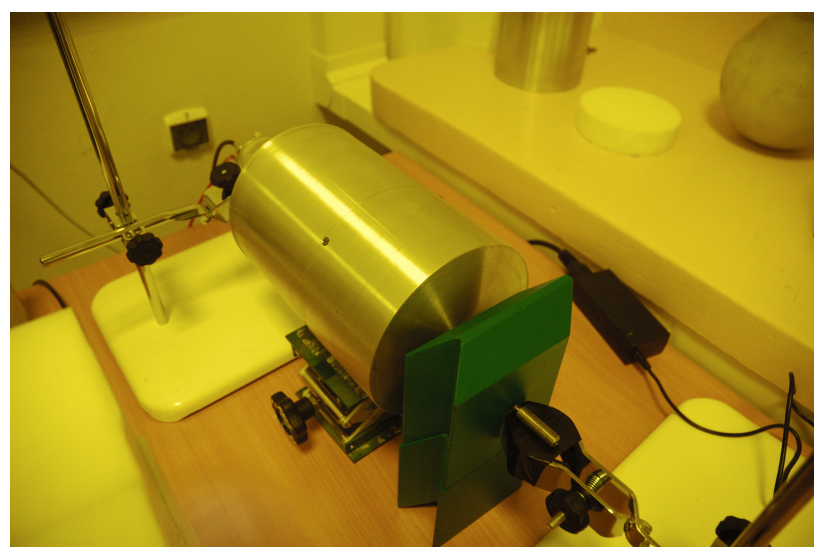

Fig. 1. Test of the prototype $\mathrm{BaF}_{2}$ detector designed at $\mathrm{NCBJ}$ with ${ }^{252} \mathrm{Cf}$ neutron source.

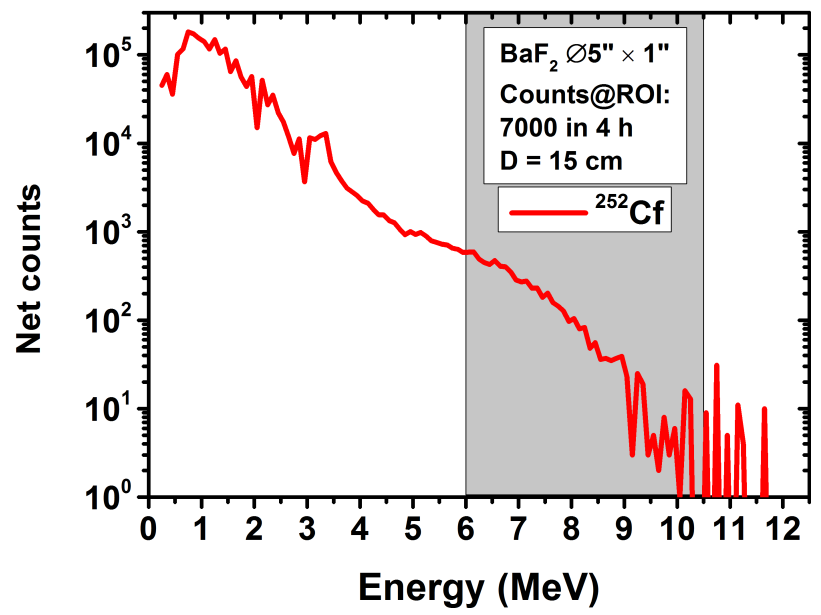

Fig. 2. The $\mathrm{BaF}_{2}$ detector response to ${ }^{252} \mathrm{Cf}$ neutron source. The $\beta$ continuum up to $10.4 \mathrm{MeV}$ is clearly seen.

$\varnothing 5 " \times 1 " \mathrm{BaF}_{2}$ based photodetector is significantly better than that for the detector presented in [3].

\section{B. Tests with $9 \mathrm{MeV}$ LINAC at SAPHIR facility}

After examination of the detector efficiency at NCBJ, the performance of depleted uranium (DU) detection were investigated at SAPHIR facility in CEA LIST Saclay, France. The test site is shown on Fig. 3. We used the commercially produced Varian Linatron M9 LINAC, emitting bremsstrahlung radiation with endpoint at $9 \mathrm{MeV}$, in order to induce photofission in DU samples. Both $\mathrm{BaF}_{2}$ and ${ }^{3} \mathrm{He}$ detectors were placed one meter from DU samples, which in turn were placed one meter from the LINAC tungsten conversion target. Measurements were performed for shielded and unshielded DU. A $5 \mathrm{~cm}$ thick $\mathrm{Pb}$ brick was used as the shielding. The Teflon in front of the $\mathrm{BaF}_{2}$ detector was used as an additional TAD material containing ${ }^{19} \mathrm{~F}$, emitting $6.1 \mathrm{MeV} \gamma$-rays. During cargo inspection it is convenient to provide as low dose as reasonable possible due to potential exposition of illegal human transportation to radiation. Thus, the LINAC beam frequency was set to $40 \mathrm{~Hz}$. Short time of inspection was introduced; irradiation lasted $10 \mathrm{~s}$, then after $1 \mathrm{~s}$ of cooling time, the acquisition with the $\mathrm{BaF}_{2}$ lasted $20 \mathrm{~s}$. Spectra were

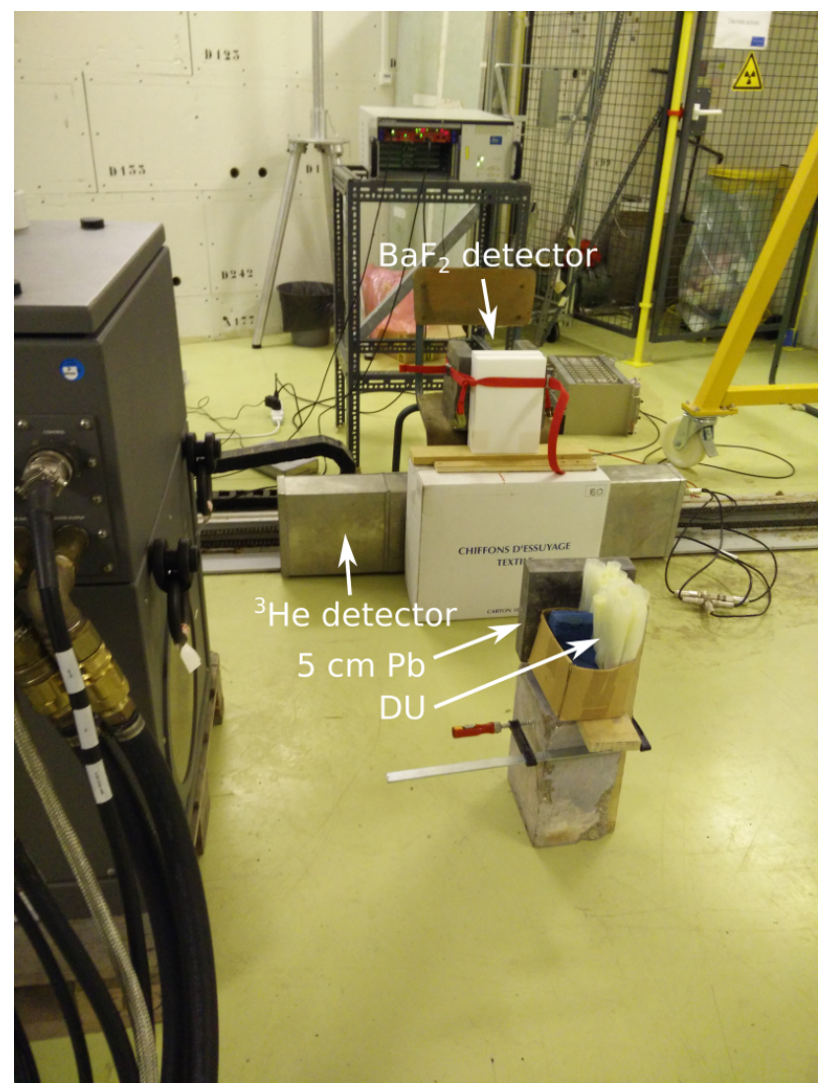

Fig. 3. Test site at SAPHIR facility, CEA LIST, France.

acquired three times, then results were summed. Summarizing, the total time of acquisition lasted $93 \mathrm{~s}$. In the case of ${ }^{3} \mathrm{He}$ detector, after single $10 \mathrm{~s}$ of irradiation, the measurement lasted $60 \mathrm{~s}$.

Fig. 4 and Fig. 5 show the obtained net spectra for the $\mathrm{BaF}_{2}$ and ${ }^{3} \mathrm{He}$ detector. The results expressed in the net number of counts were presented in Table I. The sensitivity of the TAD technique is definitely lower than the detection of delayed neutrons after irradiation with LINAC. The decrease of the

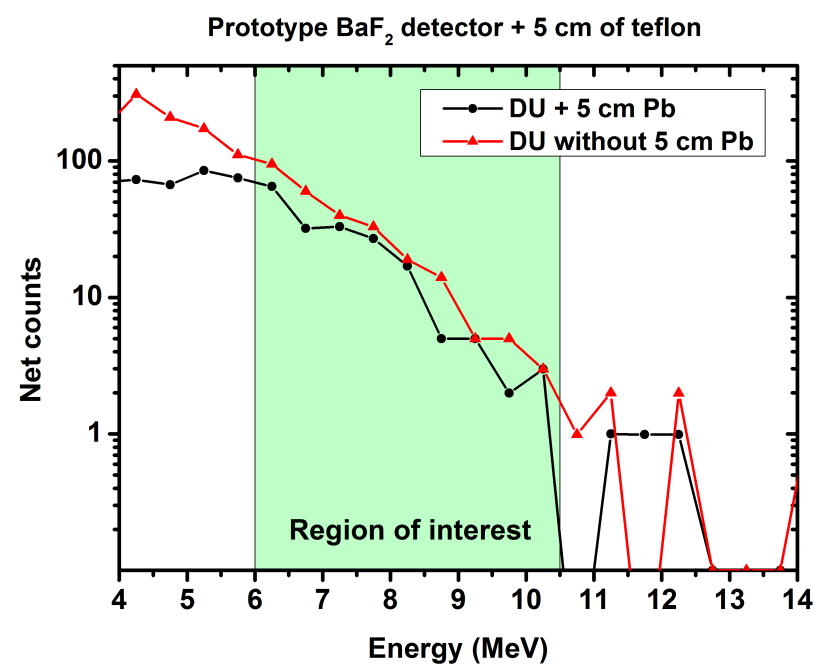

Fig. 4. Net spectra obtained from the $\mathrm{BaF}_{2}$ detector after three irradiations and counts summing. Data were binned with step of $500 \mathrm{keV}$. 


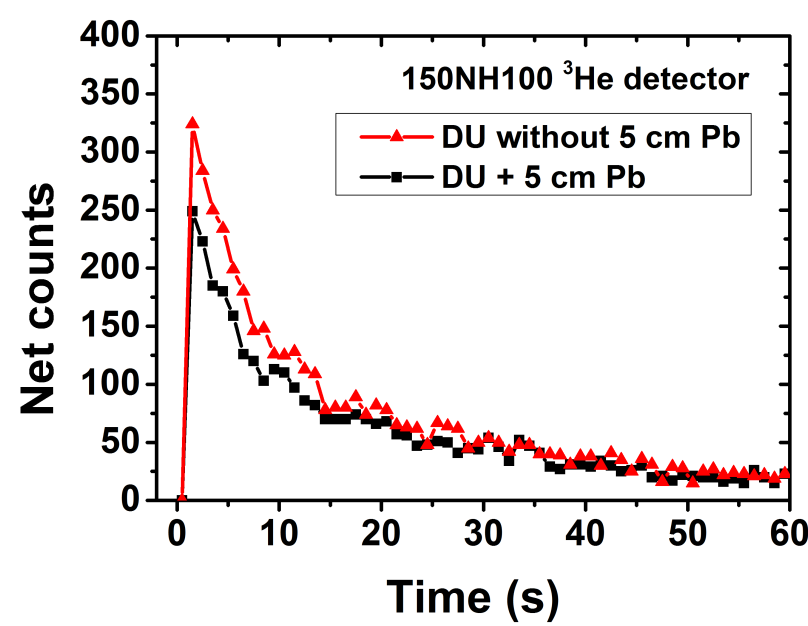

Fig. 5. The net counts registered with the ${ }^{3} \mathrm{He}$ detector in the time scale limit set to $60 \mathrm{~s}$.

TABLE I

NUMber of NeT COUNTS REgISTERED WITH THE BAF 2 AND ${ }^{3} \mathrm{HE}$ DETECTORS.

\begin{tabular}{ccc}
\hline Detector & Size & Counts \\
\hline $\mathrm{BaF}_{2}+5 \mathrm{~cm} \mathrm{~Pb}$ & $\varnothing 5 " \times 1 "$ & $189 \pm 14$ \\
$\mathrm{BaF}_{2}$ & $\varnothing 5 " \times 1 ”$ & $275 \pm 17$ \\
${ }^{3} \mathrm{He} 150 \mathrm{NH} 100+5 \mathrm{~cm} \mathrm{~Pb}$ & $\varnothing 1 \mathrm{~m} \times 1 ”$ & $3600 \pm 60$ \\
${ }^{3} \mathrm{He} 150 \mathrm{NH} 100$ & $\varnothing 1 \mathrm{~m} \times 1 ”$ & $4450 \pm 67$ \\
\hline
\end{tabular}

sensitivity of shielded DU detection is rather similar for these two techniques and varies between $20-35 \%$. However, the worldwide shortage of ${ }^{3} \mathrm{He}$ caused significant price increase of ${ }^{3} \mathrm{He}$ gas detectors. Thus, the $\mathrm{BaF}_{2}$ detector can be considered as an alternative solution offered for considerable price.

\section{CONCLUSION}

The prompt neutron detection efficiency of the $\mathrm{BaF}_{2}$ scintillator is significantly lower in comparison with the sensitivity of the $1 \mathrm{~m}$ long ${ }^{3} \mathrm{He}$ detector, used for delayed neutron detection. However, it is important to point out that the ${ }^{3} \mathrm{He}$ detector is much more expensive than $\varnothing 5 " \times 1 " \mathrm{BaF}_{2}$ detector used in the present study. Thus, comparison tests with alternative delayed neutron detectors as well as detector geometry optimization will be continued.

\section{ACKNOWLEDGMENT}

This project has received funding from the European Union's Horizon 2020 research and innovation programme under grant agreement No 653323. Disclaimer: This text reflects only the author's views and the Commission is not liable for any use that may be made of the information contained therein.

\section{REFERENCES}

[1] T. Gozani et al., "Neutron threshold activation detectors (TAD) for the detection of fissions," Nuclear Instruments and Methods in Physics Research Section A: Accelerators, Spectrometers, Detectors and Associated Equipment, vol. 652, no. 1, pp. 334-337, 2011, Symposium on Radiation Measurements and Applications (SORMA) XII 2010. [Online]. Available: http://www.sciencedirect.com/science/article/pii/S0168900211000805
[2] J. Stevenson et al., "Linac based photofission inspection system employing novel detection concepts," Nuclear Instruments and Methods in Physics Research Section A: Accelerators, Spectrometers, Detectors and Associated Equipment, vol. 652, no. 1, pp. 124-128, 2011. [Online]. Available: http://www.sciencedirect.com/science/article/pii/S0168900210018218

[3] T. Gozani, "The investigation of fast neutron Threshold Activation Detectors (TAD)," in $2^{\text {nd }}$ Iinternational Workshop on Fast Neutron Detetctors and Applications, November 6-11, 2011, Ein Gedi, Israel, 2012.

[4] P. Sibczynski et al., "Verification of threshold activation detection (TAD) technique in prompt fission neutron detection using scintillators containing ${ }^{19} \mathrm{~F}, "$ Journal of Instrumentation, vol. 10, no. 09, p. T09005, 2015. [Online]. Available: http://stacks.iop.org/17480221/10/i=09/a=T09005

[5] M. Hamel, P. Sibczynski et al., "A fluorocarbon plastic scintillator for neutron detection: Proof of concept," Nuclear Instruments and Methods in Physics Research Section A: Accelerators, Spectrometers, Detectors and Associated Equipment, vol. 768, pp. 26-31, 2014. [Online]. Available: http://www.sciencedirect.com/science/article/pii/S0168900214010407

[6] D. J. Loaiza, "High-efficiency ${ }^{3} \mathrm{He}$ proportional counter for the detection of delayed neutrons," Nuclear Instruments and Methods in Physics Research Section A: Accelerators, Spectrometers, Detectors and Associated Equipment, vol. 422, no. 1-3, pp. 43 - 46, 1999. [Online]. Available: http://www.sciencedirect.com/science/article/pii/S0168900298010596

[7] F. Carrel et al., "New experimental results on the cumulative yields from thermal fission of and and from photofission of and induced by bremsstrahlung," IEEE Transactions on Nuclear Science, vol. 58, no. 4, pp. 2064-2072, Aug 2011. 